\title{
LA IMPORTANCIA DE LA INNOVACIÓN EN EL PRODUCTO PARA GENERAR POSICIONAMIENTO EN LOS JÓVENES*
}

\author{
WILSON GIRALDO PÉREZ** \& MARÍA CRISTINA OTERO GÓMEZ**** \\ UNIVERSIDAD DE LOS LLANOS
}

Recibido/Received/Recebido: 20/04/2016 - Aceptado/ Accepted/Aprovado: 19/08/2016

\begin{abstract}
Resumen
Este artículo de investigación financiado por la Universidad de los Llanos presenta, de forma jerarquizada los factores de innovación que los consumidores aceptan como válidos para mejorar los procesos de posicionamiento y compra de productos que se ofrecen en el mercado. En este caso, los consumidores son jóvenes, hombres y mujeres, con edades que oscilan entre 16 y 24 años, residentes en Villavicencio (Colombia) quienes participaron del estudio cuantitativo. Así se planteó como hipótesis que existe una relación positiva entre las características demográficas del consumidor (edad y nivel socioeconómico) y la innovación del producto; los resultados evidencian que este grupo etario, mayoritariamente, se muestra sensible al análisis económico, puesto que gran parte de ellos dependen financieramente de un adulto y eso limita sus posibilidades de compra y consumo. En orden de importancia jerárquica, para los encuestados, la mayor calificación la obtuvieron los factores de: valor de uso y calidad percibida del producto y la menor calificación corresponde al precio de estos, finalmente no se evidencia la existencia de correlación alguna asociada a los elementos demográficos de los jóvenes de la ciudad y los factores de innovación.
\end{abstract}

Palabras clave: Innovación; Marketing; Sociología económica; Consumidor.

\section{PRODUCT INNOVATION IMPORTANCE IN ORDER TO GENERATE POSITIONING IN YOUNG PEOPLE}

\begin{abstract}
This research article, funded by Universidad de los Llanos, presents in a hierarchical way the innovation factors that consumers accept as valid to improve the processes of positioning and purchase of products offered in the market. In this case, consumers are young, male and female, ranging in
\end{abstract}

\footnotetext{
Artículo resultado de la investigación científica y tecnológica "Determinación de los factores de innovación que el consumidor valora como estrategias de posicionamiento y desarrollo de mercados" financiada por la Universidad de los Llanos mediante la modalidad de recuperación contingente durante la vigencia 2013-2014.

* Profesor Auxiliar de la Facultad de Ciencias Económicas en la Universidad de los Llanos. Administrador de Empresas de la Corporación Universitaria del Meta, Esp. en Gerencia de Mercadeo de la Corporación Universitaria del Meta, Esp. en Psicología del Consumidor de la Fundación Universitaria Konrad Lorenz, Magíster en Mercado de la Universidad de Manizales, Estudiante de Doctorado en Marketing de la Universidad de Valencia. Miembro del grupo de investigación Dinámicas de Consumo. wgiraldo@ unillanos.edu.co tel: 3013981351 Villavicencio - Colombia

*** Profesora Asistente de la Facultad de Ciencias Económicas, Universidad de los Llanos. Profesional en Comercio Internacional de la Universidad Antonio Nariño, Esp. en Derecho Comercial y Financiero de la Universidad Católica de Colombia, Esp. en Gerencia de Proyectos de la Universidad del Tolima, Magíster en Mercado de la Universidad de Manizales, Estudiante de Doctorado en Marketing de la Universidad de Valencia. Coordinadora Grupo de investigación Dinámicas de Consumo. motero@unillanos.edu.co tel: 3017417841 Villavicencio - Colombia
} 
age from 16 to 24 years old, residents of Villavicencio (Colombia) who participated in the quantitative study. Thus, it was hypothesized that there is a positive relationship between the demographic characteristics of the consumer (age and socioeconomic level) and product innovation. The results show that this age group, for the most part, is sensitive to the economic analysis, since many of them depend financially on an adult and that limits their purchasing and consumption possibilities. In order of hierarchical importance, for the respondents, the highest score was obtained by the factors of: use value and perceived quality of the product; the lower rating corresponds to the price of these; finally, there is no evidence of correlation associated with the city's youth demographic elements and factors of innovation.

Keywords: Innovation; Marketing; Economic sociology; Consumer.

\title{
A IMPORTÂNCIA DA INOVAÇÃO NO PRODUTO GERADOR DE POSICIONAMENTO ENTRE OS JOVENS
}

\begin{abstract}
Resumo
Este artigo de pesquisa financiado pela Universidade de Los Llanos apresenta, de forma hierarquizada, os fatores de inovação que os consumidores aceitam como válidos para melhorar os processos de posicionamento e compra de produtos oferecidos no mercado. Neste caso, os consumidores são jovens, homens e mulheres, com idades entre 16 e 24 anos, residentes de Villavicencio (Colômbia) e participantes do estudo quantitativo. Assim, se propôs como hipótese que existe uma relação positiva entre as características demográficas do consumidor (idade e nível socioeconômico) e a inovação do produto. Os resultados evidenciam que este grupo etário, na sua maioria, se mostra sensivel à análise econômica, já que grande parte depende financeiramente de um adulto $e$ isso limita suas possibilidades de compra e consumo. Em ordem de importância hierárquica, para os interrogados, os fatores de valor de uso e qualidade percebida do produto obtiveram a maior qualificação e o preço destes corresponde a menor qualificação. Finalmente, não se evidência a existência de correlação alguma associada aos elementos demográficos dos jovens da cidade e os fatores de inovação.

Palavras chave: Inovação; Marketing; Sociologia econômica; Consumidor.
\end{abstract}

Giraldo, W. \& Otero, M. (2017) La importancia de la innovación en el producto para generar posicionamiento en los jóvenes. En: Revista de la Facultad de Ciencias Económica: Investigación y Reflexión. rev.fac.cienc.econ, XXV (2), DOI: https://doi.org/10.18359/rfce.3072

JEL: M310, Z130.

\section{Introducción}

En los negocios, el pensamiento durante la época industrial del siglo XX era bastante claro y centrado en el precio como atributo para determinar la compra de un producto, así lo afirma Valdés (2002) "si el consumidor quería una mayor cantidad de productos a su alcance y la verdadera competencia se centraba en ofrecerlos al menor precio posible, entonces las empresas se enfocaron en la maximización del rendimiento de sus procesos productivos" (p.87). En el siglo XXI la situación es otra, con una oferta global que supera ampliamente la demanda, el principio básico del Mercadeo aborda al consumidor como un ser más complejo en la toma de decisiones, el cual escoge los bienes y servicios que mejor satisfacen sus necesidades, deseos y expectativas; Silverstein (2007) define esta combinación dada en la toma de decisiones como: escala de beneficios, "una auténtica diferencia técnica lleva a una ventaja en los resultados funcionales que puede acabar teniendo como resultado una 
respuesta emocional positiva, el apego al producto" (p.18) estableciendo así consumidores diferenciados según su intención de compra.

Por ello, es necesario conocer los diferentes grupos de consumidores actuales o potenciales con fin de desarrollar estrategias diferenciadas y adecuadas para cada segmento según sus propias escalas de beneficios, que permitan el posicionamiento de las empresas, los productos o las marcas, contribuyendo de esta forma al desarrollo de los mercados, pues tal como lo afirma David (1988) "la utilización de los conceptos y técnicas de gerencia estratégica pueden dar lugar a numerosos beneficios. Ante todo, dicho proceso permite que una organización esté en capacidad de influir en su medio, en vez de reaccionar a él" (p.46). Esta visión es compartida con Kalish (2008), quien considera que el reto más importante al que se enfrentan las compañías de productos de consumo masivo radica en el cambio del consumidor, puesto que éste comparándolo con el siglo anterior ha disminuido su edad para la toma de decisiones, y ha aumentado su capacidad de gasto; esta capacidad extra le permite especialmente a las nuevas generaciones exigir en el mercado productos más innovadores.

Estos consumidores que demandan productos más innovadores y se encuentran dispuestos a comprarlos se identifican como early adopters y según Kamakura, Kossar \& Wedel (2004) son importantes en el desempeño de las empresas puesto que con ellos, la empresa garantizará un retorno más rápido de las inversiones realizadas en el desarrollo de los nuevos productos que son lanzados al mercado.

Contextualizando los anteriores preceptos teóricos con los hallazgos para el tejido empresarial de Villavicencio, el Plan regional de competitividad del Meta (Comisión regional de competitividad del Meta, 2008) determinó que las empresas locales tienen una baja capacidad empresarial, y sus grandes retos se centran en mejorar la movilidad y participación en los mercados y ampliar su oferta productiva hacia mercados nacionales $e$ internacionales, con un proceso de transformación productiva sustentado en la innovación.
De esta forma el grupo de investigación Dinámicas de consumo, presenta los resultados de la investigación "determinación de los factores de innovación que el consumidor valora como estrategias de posicionamiento y desarrollo de mercados" financiada por la universidad de los llanos, la cual se centró en determinar en primera instancia que tipo de innovación (de las planteadas por la OCDE) era mejor evaluada por los consumidores jóvenes, posteriormente se establecieron los factores asociados a ese tipo de innovación mejor evaluada que ayudan al planteamiento de estrategias de posicionamiento y participación en los mercados, puesto que es éste uno de los grandes retos evidenciado en los hallazgos del gobierno municipal, y del cual se carecen de estudios aplicados para la ciudad. Entre sus resultados más significativos la investigación permite afirmar que el tipo de innovación mejor evaluada por los jóvenes es la referida al producto, y para el $78 \%$ de las mujeres es importante para favorecer el posicionamiento, que ésta innovación sea fácilmente perceptible y facilite el uso del producto, mientras que para los hombres el porcentaje llega al $70 \%$ en este mismo análisis.

\section{Materiales y métodos}

En el desarrollo de la investigación cuantitativa, se recolectaron datos mediante encuestas aplicadas en Villavicencio (Colombia) de forma personal a 384 jóvenes y adultos jóvenes de colegios y universidades, quienes fueron seleccionados como informantes voluntarios mediante un muestreo No probabilístico, la muestra incluyó personas entre 16 y 24 años, de distintos niveles socioeconómicos de la ciudad y de sexo masculino y femenino distribuidas como se presenta en la tabla 1 en cuotas iguales.

Tabla 1. Distribución de grupos para la muestra

\begin{tabular}{|l|l|l|}
\hline & NSE* Bajo & NSE Alto \\
\hline Hombre menor de edad & $12,5 \%$ & $12,5 \%$ \\
\hline Hombre mayor 18 y menor de 24 años & $12,5 \%$ & $12,5 \%$ \\
\hline Mujer menor de edad & $12,5 \%$ & $12,5 \%$ \\
\hline Mujer mayor 18 y menor de 24 años & $12,5 \%$ & $12,5 \%$ \\
\hline
\end{tabular}

*Nivel socioeconómico

Fuente: Elaboración de los autores. 
El instrumento inicial para la investigación fue diseñado con 19 preguntas y sometido a una prueba piloto la cual se procesó y calcularon los indicadores de fiabilidad y validez; el instrumento se ajustó y probó nuevamente en una segunda prueba pilo- to, para quedar, finalmente, con 16 preguntas de escala Likert correspondientes a la variable innovación investigada a través de cuatro (4) factores; los indicadores finales se presentan en las tablas 2 y 3.

Tabla 2. Indicadores de validación del instrumento Factor por Factor

\begin{tabular}{|l|c|c|l|}
\hline \multicolumn{1}{|c|}{ Factores } & Alfa de Cronbach & $\begin{array}{c}\text { Validez Convergente - Fiabilidad } \\
\text { Compuesta }\end{array}$ & $\begin{array}{l}\text { Validez Discriminante - Test de intervalo } \\
\text { de confianza (límite superior) }\end{array}$ \\
\hline Precio & 0,71 & 0,74 & $\mathrm{f} 1-\mathrm{f} 2=0,86 \mathrm{f} 1-\mathrm{f} 3=0,48 \mathrm{f} 1-\mathrm{f} 4=0,525$ \\
\hline Valor de Uso & 0,75 & 0,75 & $\mathrm{f} 2-\mathrm{f} 3=0,69 \mathrm{f} 2-\mathrm{f} 4=0,98$ \\
\hline Lealtad & 0,73 & 0,73 & $\mathrm{f} 3-\mathrm{f} 4=0,68$ \\
\hline Calidad Percibida & 0,76 & 0,79 & \\
\hline
\end{tabular}

Fuente: Elaboración de los autores.

Tabla 3. Indicadores de bondad de ajuste del instrumento

\begin{tabular}{|l|l|l|}
\hline \multicolumn{1}{|c|}{ Ajuste } & \multicolumn{1}{|c|}{ Indicador } & \multicolumn{1}{c|}{ Resultado } \\
\hline \multirow{3}{*}{ Absoluto } & Chi Cuadrado & 356,08 \\
\cline { 2 - 3 } & Grados de libertad & 98 \\
\cline { 2 - 3 } & Standardized Root Mean Square Residual (SRMR) & 0,069 \\
\hline Parsimonioso & Root Mean Square of Error Aproximation (RMSEA) & 0,080 \\
\hline Incremental & Comparative Fit Index & 0,084 \\
\hline
\end{tabular}

Fuente: Elaboración de los autores.

Los factores correspondientes a la variable innovación fueron jerarquizados y analizados según la calificación emitida por los jóvenes, y se clasificaron de forma descendente con base en la siguiente valoración cualitativa y cuantitativa (a partir de la media de las calificaciones): Prioritarios aquellos factores cuya calificación promedio fue superior a 3 , importantes para los que la calificación promedio se encontraba entre 3 y 2,6, relevantes los factores con una calificación entre 2,5 y 2, y finalmente los irrelevantes con calificación menor a 2.
Los datos recolectados se procesaron en los software SPSS y EQS, para determinar las propiedades del instrumento y se realizaron los cálculos de las tablas de frecuencias, las pruebas Chi cuadrado, los coeficientes de correlación Tau de Kendall se realizaron para determinar si existía algún nivel de asociatividad entre las variables: edad y nivel de importancia de los factores de innovación en producto, o, nivel socioeconómico y nivel de importancia de los factores de innovación en producto, todas ellas con un nivel de significancia de 0,05. 


\section{Revisión de literatura}

La innovación tiene un papel importante para el tejido empresarial y el entorno tanto de los negocios como del marketing, pues tal como lo afirman Helfat et al. (2007) este concepto hace parte de las capacidades dinámicas de la empresa y ella le permite crear, extender o modificar su base de recursos intencionalmente (p. 4), pero esa intencionalidad de la empresa de ampliar su oferta mediante uno de estos tres caminos queda circunscrita a las expectativas de quienes utilizarán en su propio beneficio esa innovación, es decir: los consumidores.

Esta influencia del consumidor y del entorno en la innovación es referida en la literatura correspondiente a este tema, afirman Moreno \& García (2014) que converge en este término una doble dimensión, por una parte innovación como sistema (Schumpeter, 1939; Nelson, 1993) y por otra, innovación como proceso (Lundvall, 1992; Nelson, 1997), bajo esta última dimensión en las organizaciones se requieren decisiones y acciones que sean determinantes, pero, estas se encuentran influenciadas por el entorno dinámico (p.111).

Así, para no generar errores interpretativos, en el presente artículo la innovación se entenderá según la siguiente definición:

Introducción de un nuevo, o significativamente mejorado: producto (bien o servicio), de un proceso productivo, de un nuevo método de comercialización o de un nuevo método organizativo, en las prácticas internas de la empresa, la organización del lugar de trabajo o las relaciones exteriores (OCDE, 2006)

A partir de la anterior definición y conformando lo planteado por Moreno y García, la innovación puede ser tomada como un proceso ligado a las capacidades internas organizacionales (tipos de innovación según la OCDE: Proceso productivo o Método organizativo) o, como un sistema que vincula las capacidades internas organizacionales con el entorno dinámico en el que se encuentra la demanda (innovación en producto, o en método de comercialización). Este artículo frente a la innovación toma una postura sistémica, pues tal como lo afirma Arnold (1998) "mientras más equivalencias reconozcamos entre organismos, máquinas, hombres y formas de organización social, mayores serán las posibilidades para aplicar correctamente el enfoque de la TGS" (p.41). Así finalmente la empresa se encuentra inmersa en un mercado, y en él desarrolla sus diferentes estrategias para posicionar un producto y luego comercializarlo.

La generación de posicionamiento en el mercado según Ries \& Trout (1992), aunque requiere un artículo, servicio, compañía o persona, no se refiere al producto en sí mismo; sino a lo que se hace con la mente de los probables clientes mediante la comunicación y, "su enfoque fundamental no es crear algo nuevo y diferente; sino revincular las conexiones que ya existen en la mente" (p.7)

Todos los productos tienen por naturaleza y basados en la literatura varias características entre las que se cuentan los atributos económicos, funcionales y emocionales, con respecto a los atributos económicos las estrategias asociadas al factor precio permiten por una parte a la empresa obtener beneficios financieros a corto plazo y por otro, se convierte en un elemento de percepción y marco de referencia para las decisiones de compra del consumidor.

Dentro de los atributos funcionales se encuentran los factores de Calidad percibida y Valor de uso, los cuales ayudan en la estrategia empresarial de posicionamiento puesto que permiten entender el quehacer del producto y su utilidad actual o futura permitiendo esa conexión en la mente del consumidor. Posición coherente con el enfoque cognitivo de las decisiones de compra propuesto por East (1997) para quien el detonante de la compra es el producto en sí mismo, y por Park et al. (1986) quienes afirman que los productos, servicios o marcas se compran de acuerdo a las necesidades que estos cubren.

Finalmente en los atributos emocionales se ubica el factor lealtad definido por Oliver (1999) como un "compromiso profundo de recompra de un producto preferido de manera consistente en el futuro", convirtiendo así este factor en tema importante para la innovación del producto y para su po- 
sicionamiento en el segmento de los jóvenes early adopters dentro del entorno dinámico (mercado).

Al analizar las características demográficas del consumidor y su relación con estos cuatro factores de innovación se buscó probar la hipótesis soportada en la literatura que analiza la relación entre un aumento en la edad y una menor intención de cambio de productos innovadores (Wood, 2004) o, de un nivel de ingresos más altos del consumidor y su mayor fidelidad por los productos que conoce (East, Harris \& Hammond, 1995); así se planteó para la presente investigación que existe una relación posi- tiva entre las características demográficas del consumidor (edad y nivel socioeconómico) y la innovación del producto.

\section{Resultados}

Los resultados presentados a continuación son aplicables para el análisis de la población joven (menor de edad y adulto joven) en la ciudad de Villavicencio Colombia, y se centran en la innovación mejor evaluada por ellos (ver imagen 1) según la tipología planteada por la OCDE que fue Innovación en Productos.

Imagen 1. Jerarquización de los tipos de innovación OCDE

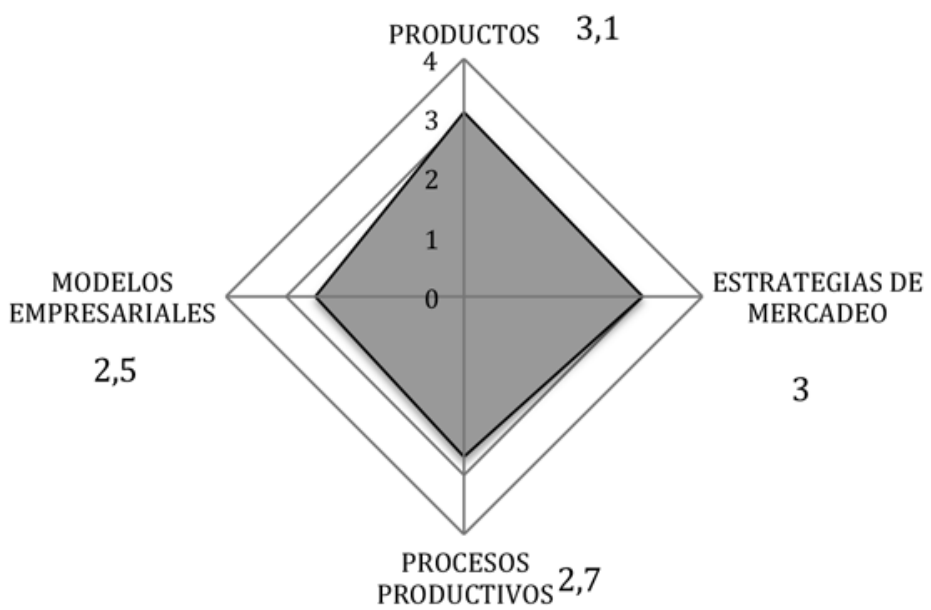

Fuente: Elaboración de los autores. 2015

La tabla 4 y la imagen 2 jerarquizan en orden descendente los factores de innovación aplicables a la innovación en producto. Una vez organizados jerárquicamente los factores se clasificaron así:

- Importantes, se ubica el $75 \%$ de los factores estudiados
- Relevantes, en esta categoría se encuentra el $25 \%$ de los factores objeto de análisis.

- Según la calificación de los jóvenes no se ubica ningún factor en los extremos de la calificación, es decir como prioritario o como irrelevante. 
Tabla 4. Calificación de los factores de innovación para las estrategias de productos según los jóvenes

\begin{tabular}{|l|l|l|l|l|}
\hline \multicolumn{1}{|c|}{ FACTORES } & \multicolumn{1}{c|}{ Calificación } & \multicolumn{1}{c|}{ Importancia } & \multicolumn{2}{c|}{ Jerarquización } \\
\cline { 1 - 4 } Innovación en valor de uso & 2,8 & $70 \%$ & Importante & \multirow{2}{*}{$75 \%$} \\
\cline { 1 - 4 } Innovación en calidad percibida & 2,8 & $70 \%$ & Importante & \\
\cline { 1 - 4 } Innovación en estrategia de lealtad & 2,6 & $65 \%$ & Importante & \multirow{2}{*}{$25 \%$} \\
\hline Innovación en precio & 2,3 & $58 \%$ & Relevante & \\
\hline
\end{tabular}

Fuente: Elaboración de los autores. 2015

Imagen 2. Jerarquización de factores de innovación para las estrategias de productos según los jóvenes

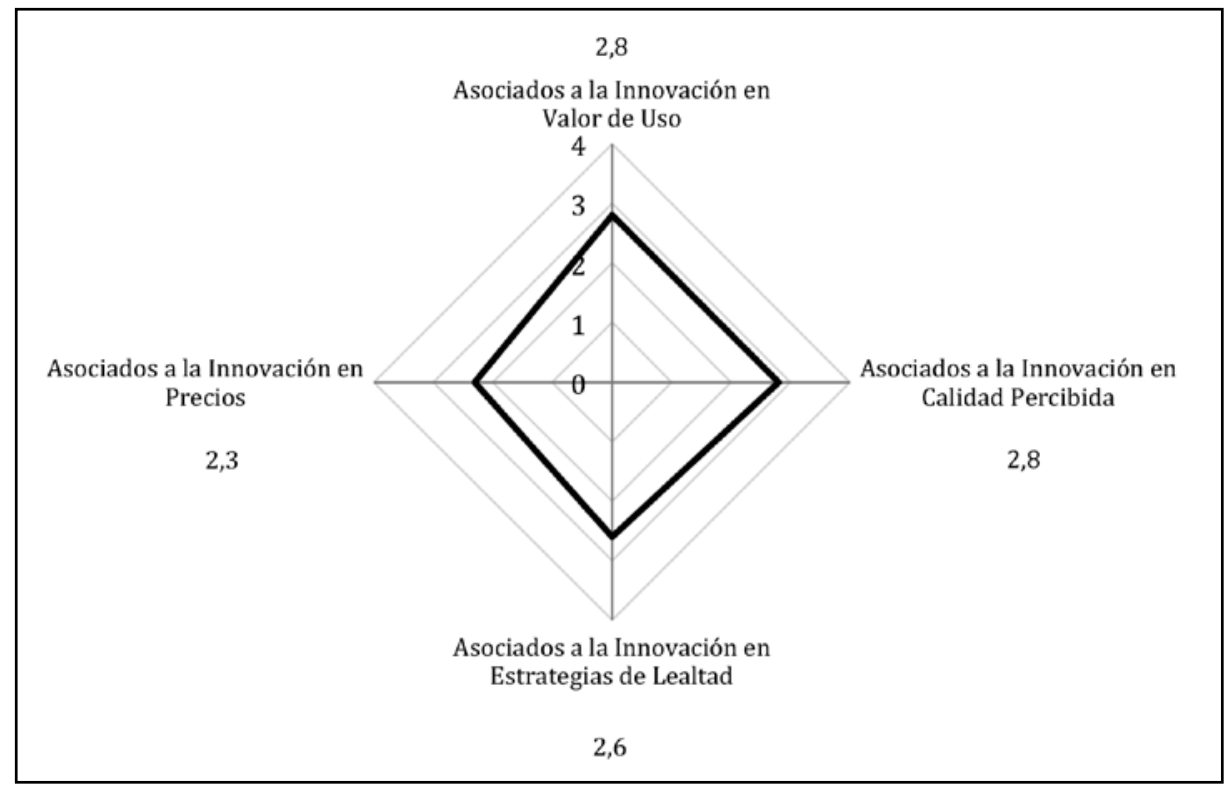

Fuente: Elaboración de los autores. 2015

Frente a la hipótesis planteada, los resultados presentados en las tablas 5 y 6 no evidencian la existencia de correlación alguna asociada a los elementos demográficos de los jóvenes de la ciudad (edad o nivel socioeconómico) y los factores de innovación, por lo tanto se rechaza la hipótesis planteada significando lo anterior que para este contexto, las variables presentan un comportamiento independiente. 
Tabla 5. Correlaciones por rango de edad y factores de innovación

\begin{tabular}{|l|l|l|l|l|l|}
\hline \multicolumn{2}{|l|}{} & $\begin{array}{c}\text { Factor Valor de Uso } \\
\text { en Innovación de } \\
\text { Producto }\end{array}$ & $\begin{array}{c}\text { Factor Calidad Percibida } \\
\text { en Innovación de } \\
\text { Producto }\end{array}$ & $\begin{array}{c}\text { Factor Lealtad } \\
\text { en Innovación de } \\
\text { Producto }\end{array}$ & $\begin{array}{c}\text { Factor Precio en } \\
\text { Innovación de } \\
\text { Producto }\end{array}$ \\
\hline \multirow{4}{*}{$\begin{array}{l}\text { Rango } \\
\text { etario }\end{array}$} & $\begin{array}{l}\text { Correlación } \\
\text { Kendall }\end{array}$ & 0,041 & $-0,057$ & $-0,009$ & $-0,03$ \\
\cline { 2 - 7 } & Sig (Bilateral) & 0,421 & 0,262 & 0,863 & 0,553 \\
\cline { 2 - 7 } & $\begin{array}{l}\text { Gri-Cuadrado } \\
\text { Grados de }\end{array}$ & 1,495 & 1,677 & 3,128 & 4,326 \\
\cline { 2 - 7 } & 3 & 3 & 3 & 3 \\
\hline
\end{tabular}

Fuente: Elaboración de los autores. 2015

Tabla 6. Correlaciones por nivel socioeconómico y factores de innovación

\begin{tabular}{|l|l|l|l|l|l|}
\hline \multicolumn{2}{|c|}{} & $\begin{array}{l}\text { Factor Valor de Uso } \\
\text { en Innovación de } \\
\text { Producto }\end{array}$ & $\begin{array}{c}\text { Factor Calidad } \\
\text { Percibida en } \\
\text { Innovación de Producto }\end{array}$ & $\begin{array}{c}\text { Factor Lealtad } \\
\text { en Innovación de } \\
\text { Producto }\end{array}$ & $\begin{array}{c}\text { Factor Precio en } \\
\text { Innovación de } \\
\text { Producto }\end{array}$ \\
\hline \multirow{4}{*}{$\begin{array}{l}\text { Nivel Socio } \\
\text { Económico }\end{array}$} & $\begin{array}{l}\text { Correlación } \\
\text { Kendall }\end{array}$ & 0,063 & $-0,105$ & $-0,019$ & 0,1 \\
\cline { 2 - 7 } & Sig (Bilateral) & 0,219 & 0,04 & 0,713 & 0,062 \\
\cline { 2 - 7 } & $\begin{array}{l}\text { Grados de } \\
\text { Libertad }\end{array}$ & 3 & 5,799 & 1,126 & 12,007 \\
\cline { 2 - 7 } & Sig (Bilateral) & 0,577 & 3 & 3 & 3 \\
\cline { 2 - 7 } & $\mathrm{N}$ & 384 & 0,122 & 0,771 & 0,007 \\
\hline
\end{tabular}

Fuente: Elaboración de los autores. 2015

Donde se encuentran evidencias de diferencias de alguna diferencia es en los resultados acerca de la importancia que le brindan a la innovación los hombres y las mujeres presentados en la tabla 7.

Tabla 7. Nivel de importancia de la innovación por género

\begin{tabular}{|l|l|l|l|l|l|}
\hline \multicolumn{3}{|c|}{ Mujeres } & \multicolumn{3}{c|}{ Hombres } \\
\hline & Casos & Porcentaje Válido & & Casos & Porcentaje Válido \\
\hline Muy importante & 102 & $53 \%$ & Muy importante & 87 & $45 \%$ \\
\hline Importante & 48 & $25 \%$ & Importante & 48 & $25 \%$ \\
\hline Poca importancia & 16 & $8 \%$ & Poca importancia & 27 & $14 \%$ \\
\hline Sin importancia & 26 & $14 \%$ & Sin importancia & 30 & $16 \%$ \\
\hline Total & 192 & $100 \%$ & Total & 192 & $100 \%$ \\
\hline
\end{tabular}

Fuente: Elaboración de los autores. 2015 


\section{Discusión de resultados}

Cuando se aborda el marketing desde los aspectos sociales y económicos, éste busca el bienestar de las partes involucradas en el proceso de intercambio de productos. Empero, este intercambio va más allá de lo estrictamente comercial, es decir que los bienes y servicios se evalúan no solo desde el valor de uso que se le otorgue a éstos, por el contrario este intercambio está cargado de simbolismos que resultan difíciles de cuantificar o medir.

Bajo esta mirada, surgen posiciones más holísticas e integradoras como la planteada por Duque (2008):

El marketing no se puede quedar en aspectos meramente instrumentales y mecánicos para su accionar. Con la antropología sociocultural se busca esclarecer y profundizar en lo estratégico e indagar las relaciones que los consumidores establecen con la publicidad, la comercialización, las relaciones públicas, el merchandising y especialmente con los objetos (productos y servicios), en los diferentes escenarios de consumo de una cultura en especial, en el marco de una clase social y un estilo de vida (p.6).

Lo anterior permite comprender que las relaciones entre consumidor y consumo se encuentran mediadas por las estrategias empresariales y la innovación es soporte de esas estrategias, Mensch (citado por Cáceres \& Aceytuno, 2008) determina que estas innovaciones aparecen de forma individual $e$ independiente en las empresas, las cuales no poseen características comunes o comparten tecnología similar con toda la industria, siendo de esta forma la innovación un resultado de los esfuerzos de los empresarios mediante investigación y desarrollo de productos. (p.139).

Para Colombia Coronado, Echeverry \& Arias (2014) encontraron en su investigación que, para 19 empresas activas en procesos de cooperación empresarial que hacen parte del Programa de Asociatividad y Desarrollo Empresarial Sectorial -PADES- todas ellas pertenecientes a la Asociación Colombiana de Medianas y Pequeñas Industrias ACOPI- el 73,68\% fue importante y realizaron me- joras en sus productos y, el 63,16\% de las empresas introdujo un nuevo producto durante el periodo estudiado a saber 2009-2012. (p. 196).

Estos datos permiten enmarcar los resultados obtenidos para Villavicencio y la relevancia que en esta ciudad también tiene el producto dentro de las estrategias empresariales así:

A. Jerarquización de los tipos de Innovación fijados por la OCDE y Eurostat, en el manual de Oslo $3^{a}$ edición del año 2005 según los jóvenes de Villavicencio:

En la imagen 1 se presentaron los resultados de esta jerarquización y ellos evidencian la existencia de una innovación que en calificación promedio de los consumidores jóvenes de Villavicencio supera a los otros tres tipos, lo que permitió determinar que el tipo más importante para el segmento estudiado según la propuesta de la OCDE es: la innovación de productos.

El resultado es concordante con los datos acerca de artículos científicos, referente a este tema, de innovación se han escrito y publicado en los últimos 50 años según la base de datos ABI/INFORM ${ }^{\mathrm{TM}} 143$ artículos que en sus palabras claves referencian este término, destacándose los últimos 5 años en los que se han producido 71 de ellos, lo que representa que el $50 \%$ del total de la literatura alrededor de este tema es reciente y refleja la tendencia mundial de estos resultados.

Al comparar lo investigado para Villavicencio en otros países, se encontró que en Chile, según el estudio de percepciones y actitudes hacia la innovación, cuando se preguntó sobre los responsables de realizar las innovaciones, el 55\% respondió que eran las personas, el 19\% afirmó que son las empresas, el $14 \%$ que las universidades y centros de estudios y el $11 \%$ que es responsabilidad del Estado (Corporación de Fomento de la Producción, 2013). Estos resultados son consistentes con los obtenidos en esta investigación, al ubicar a la empresa en niveles secundarios cuando se trata la innovación como un proceso bien sea productivo, o de modelo empresarial. 
En otro contexto también internacional, con un estudio realizado en Uruguay, en 2008, a personas adultas entre las que se encontraban jóvenes con edades entre 16 y 24 años de edad, con el fin de identificar cuál es la primera palabra en la que pensaban cuando se hablaba de innovación; se encontró que la asociaban con: algo nuevo $37 \%$, progreso $16 \%$, equipos y maquinaria $9 \%$, conocimiento $5 \%$ y otras 9\% (Agencia Nacional de Investigación e innovación, 2008). De esta forma, también en Villavicencio los jóvenes aceptan "algo nuevo" en el producto representado en lo estrictamente nuevo o significativamente mejorado de los bienes o servicios disponibles en el mercado, puesto que es lo que finalmente les interesa intercambiar y así satisfacer sus necesidades.

A nivel nacional, un estudio realizado en Barranquilla, acerca de la compra impulsiva y el materialismo en los jóvenes, permitió establecer que la categoría ropa se constituye en el producto por excelencia en las compras, ya que genera satisfacción al momento de su uso, le permite proyectar una imagen frente a los otros y es tomada como criterio de cambio cuando se usa ropa actualizada, innovadora o de moda (Luna, Puello \& Botero, 2004). Así la ropa para los barranquilleros se convierte en lo usable y por ello al igual que en Villavicencio, es la innovación que encuentran en el producto lo más valorado por este grupo etario de referencia.

Por lo anterior se puede interpretar a los jóvenes como, aquellos consumidores que consideran innovador lo que es tangible o lo que efectivamente reciben del mercado como satisfactores de necesidades.

De este modo al jerarquizar los tipos de innovación según la OCDE, los modelos empresariales, el proceso productivo y la estrategia que utilice la empresa, aunque son importantes para que el producto innovador llegue a manos del consumidor, son innovaciones que suceden al interior de la empresa y para los jóvenes no revisten la misma importancia puesto que estas no pueden ser vistas y tangibilizadas por ellos, determinando que la innovación en producto es la que ocupa el primer lugar y por tanto la más importante para el grupo estudiado.

\section{B. Jerarquización de los factores de la innova- ción en Productos.}

Para explicar la innovación de producto en los jóvenes, se les solicitó evaluar una serie de afirmaciones planteadas en el instrumento que hacen parte del concepto estudiado, ellas son jerarquizadas de forma descendente y presentadas en la tabla 4 y la imagen 2. En ellos puede observarse que el valor de uso y la calidad percibida son los factores con más alta calificación, y que a diferencia de lo planteado en época industrial, el precio de producto (atributo económico) en el siglo XXI ha perdido relevancia para los consumidores jóvenes, al obtener la calificación más baja de los factores evaluados y el factor asociado a las estrategias de lealtad de marca (atributo emocional) no ocupa un lugar primordial para los jóvenes.

Lo anterior permite a los autores entender que referente a la innovación y significación de los productos este segmento en la actualidad se comporta según lo planteado por Silverstein en lo referente a la escala de beneficios pues los artículos comprados deben tener atributos funcionales (usabilidad) pero deben satisfacer sus expectativas (calidad), en esta línea Jiménez \& Ramos (2007) argumentan que, para los jóvenes Españoles, la personalización en la categoría de tecnología cobra gran importancia, pues ellos "requieren continuas novedades para reafirmar su personalidad y sentirse más importante dentro del grupo" siendo esta su forma de escalar en los beneficios buscados del producto, así producto y escala deben estar contextualizados en una marca que, finalmente, permite sustituir la relación abstracta entre consumidores y productos, por una relación entre unos gustos por productos de marca a los que confieren un significado sus diferentes identidades culturales (Bordieu, 1998), sin que esta sea lo suficientemente poderosa como para ubicarse en primer lugar entre los jóvenes del siglo XXI y basar en el factor marcario su decisión de consumo.

Los factores importantes en conjunto representan un $75 \%$ del total y hacen parte de esta categoría: el valor de uso, la calidad percibida y las estrategias de lealtad de marca; ello significa que los jóvenes 
estudiados desean que la empresa proveedora de los productos haga más que comunicarles los beneficios de éste, desean sentirlos a su alcance, situación explicable desde la realidad que los jóvenes debido a su limitación de recursos disponibles buscan la maximización de los beneficios por el costo que pagan por los productos, y por ello compran y recompran las marcas más reconocidas a las que le atribuyen por una parte, en su propia valoración subjetiva, mayor calidad; y por otra parte, si todos los miembros de su grupo compran y consumen determinada marca, ella adquiere, vía masificación de su uso, un aumento en la percepción de su calidad para todos sus iguales.

Las limitaciones económicas que continúan presentes en el análisis de la jerarquización de estos factores se hacen más evidentes generalmente durante la etapa de estudios o aun siendo profesionales cuando no cuentan con un trabajo estable, por ende buscan la satisfacción de sus necesidades pero, de una forma balanceada entre precio-marca-calidad. Otras investigaciones orientadas a comprender las decisiones de compra en los jóvenes, permiten encontrar puntos afines. Por ejemplo, Kuster, Aldás, Santos \& Vila (2010), en un estudio comparativo realizado en Europa, afirman que las preferencias de compra en el segmento joven varían, de tal forma que en Italia se inclinan por nuevos colores y envases modernos; en España, valoran ampliamente la utilidad y funcionalidad del producto siendo estos jóvenes similares a los de Villavicencio; mientras que en Francia predomina el consumo de bienes asociados al comercio justo.

Siendo así, se configura este segmento de consumidores en Villavicencio (Colombia) como un grupo más predispuesto a la compra con un predominio de las características funcionales de los productos, sobre las emocionales.

Los factores relevantes representan el $25 \%$ del total de los factores evaluados y se debe prestarse atención a ellos pues son los que permiten operativizar y soportar los tres factores anteriores. Los jóvenes de la ciudad califican en promedio a las estrategias ligadas al manejo de los precios del producto dentro de esta categoría, explicado, nuevamente en parte, por la dependencia económica, pues al existir una promoción para una categoría que al joven le resulte atractiva, estará sujeto a la disponibilidad de esa capacidad de compra del adulto quien provee el dinero para efectuar la transacción, muchas veces sin la oportunidad de aprovecharla, razones que convierten a las promociones y en general a las estrategias ligadas a este atributo económico, en el que genera menos posicionamiento y por tanto menor oportunidad de desarrollo dentro de este segmento de mercado.

Cuando el precio es manejado en plataformas digitales, los jóvenes son una fuerza viva muy poderosa en número, necesidades y tamaño proporcional de la pirámide poblacional, pero, no lo son en acceso al sistema financiero, debido a que no poseen una fuente de ingresos fija, o tarjetas de crédito que les permitan realizar estas transacciones.

Por ello, como lo afirman Otero \& Giraldo (2014), "las compras por internet a cualquier edad y en cualquier nivel socioeconómico sugieren un saber y un tener. Por una parte un "saber" en el manejo de plataformas donde realizar las compras en internet, y un manejo aceptable del idioma inglés. Y por otro lado un "tener" tarjeta de crédito disponible para efectuar la compra, aparatos tecnológicos y conectividad a internet para realizar la transacción". Conjugación de situaciones que no son las más frecuentes de encontrar en el grupo de jóvenes estudiados.

C. Correlaciones entre la importancia de la innovación y las variables demográficas edad y nivel socioeconómico.

Opuesto a Schumpeter (1946 y 1968), quien plantea la innovación como un resultado empresarial que se puede convertir en un medio para lograr monopolizar el mercado, aparecen 50 años después autores como Cohen \& Levin (1989) quienes exponen que es la demanda y no la oferta la que dirige el avance y las innovaciones; conocida como la tesis del empuje de la demanda, es ella la que propone un abanico de posibilidades a las organizaciones, las cuales seleccionan las que pueden convertir en oportunidades de negocio. Este planteamiento ha 
sido muy criticado, puesto que subvalora la importancia de la tecnología, pero es tomado en el presente artículo como referente debido a que reconoce al consumidor como el iniciador y transformador del proceso organizacional, pues las empresas deben ajustarse a los gustos y necesidades cambiantes del consumidor del siglo XXI, especialmente del más joven. Malaver \& Vargas (2004) también evidencian para Colombia este planteamiento y afirman que en el ámbito externo, la fuente principal de las innovaciones son los clientes. (p. 30).

Por ello se analizó si un aumento en la edad de los jóvenes significaba una asociación con la importancia en algún factor de innovación (Tabla 5) $y$ se puede inferir que, divididos en dos grupos de edad, el primero de 16 a menos de 18 años denominado "menor de edad" y el segundo de 18 a 24 "adulto joven", ninguna correlación es lo suficientemente fuerte para considerar que el aumento de la edad produce un aumento en la importancia de los factores sometidos a estudio, siendo solo el factor Valor de uso el que obtiene un dato positivo pero tendiente a cero, evidenciando la independencia en el comportamiento de estas dos variables.

Al hacer el análisis por niveles socioeconómicos, en la tabla 6 , el grupo se dividió en jóvenes pertenecientes al NSE bajo y al medio-alto y, tal como sucedió en la división anterior, se prueba que por niveles socioeconómicos los valores del coeficiente de Tau de Kendall no reflejan ninguna correlación fuerte para considerar que un aumento en el nivel socioeconómico de los jóvenes signifique también un aumento de importancia en algún factor de innovación; inclusive en los factores: Calidad percibida y lealtad de marca con fuerza cercana a cero, la dirección de la correlación es negativa.

Los datos anteriores no permiten aceptar como válida la hipótesis planteada y soportada en las posturas de Wood (2004) y East, Harris \& Hammond (1995) significando que el nivel de importancia de los factores de innovación se presenta en el grupo etario jóvenes en general y su respuesta no obedece a ninguna división demográfica del segmento.

\section{Diferencias encontradas en el segmento}

Para finalizar el análisis y al tener en cuenta que el total de la categoría jóvenes se convierte en un potencial dinamizador de la demanda y en fuente de innovaciones para las empresas, se realizó el análisis a nivel de género y se encontró que divididos así, si existe variación porcentual frente a la mayor importancia de la innovación sobre las otras estrategias, puesto que, para las mujeres, esta llega al 78\%, frente al 70\% entre los hombres; estos datos son comparables también en otros contextos latinoamericanos como en Chile, país en el cual durante el 2006 se aplicó una encuesta a 6.000 estudiantes universitarios dentro de los cuales el $77 \%$ correspondió a jóvenes entre 18 y 22 años, con el objetivo de conocer las nuevas transformaciones culturales, prácticas y percepciones, se pudo identificar en sus resultados que la actitud frente a la ciencia y las innovaciones tecnológicas reflejaron una valoración radicalmente positiva que, por lo demás, es más fuerte en las mujeres $(70,1 \%)$ que en los hombres $(65,4 \%)$, dejando así de lado el mito de una insensibilidad relativa femenina frente a un tema que otrora fue considerado como propio de un ámbito masculino (Baeza, 2006).

Los datos anteriores, coinciden para los países y evidencian que, para el género femenino, la ciencia y la innovación en sus diferentes ámbitos son sinónimos de modernidad y progreso, y no tenerlas puede ser entiendo por los jóvenes como sinónimo de atraso de la colectividad a la que se pertenece.

\section{Conclusión}

Respecto a la jerarquización de los factores que permiten la innovación en producto y la importancia dada a estos para generar posicionamiento, puede ser analizada como un todo incluidos los menores de edad y los adultos jóvenes de todos los niveles socioeconómicos de la ciudad. Lo que significa que los resultados pueden ser extrapolables a este grupo etario siendo adecuado hablar genéricamente de Jóvenes. Puesto que según los resultados anteriores, no se evidencian diferencias significativas que ameriten una micro segmentación, y la única diferencia porcentual hallada fue por géneros, la cual es menor al $10 \%$. 
Por ello, para generar posicionamiento en los jóvenes de Villavicencio, se debe trabajar en el aumento de los atributos funcionales expresados en los factores valor de uso y la calidad percibida de los productos que fueron calificados por ellos y clasificados por los autores en la categoría de importantes; ello acompañado de mensajes detallados acerca de los beneficios que obtendrán por la compra de una marca en concreto destacando los atributos emocionales en su uso.

Es decir que la compra en este segmento no es un efecto compulsivo o irracional; por el contrario, debido a sus limitaciones de recursos, la compra y el consumo para estos jóvenes se convierte en la oportunidad de satisfacer sus necesidades y en una reivindicación con su grupo etario y social, mediante la importancia que le dan las empresas y el poder que le otorga el uso de una determinada marca a los consumidores jóvenes.

\section{Referencias}

Agencia Nacional de Investigación e Innovación. (2008). Encuesta de percepción pública sobre ciencia, tecnología e innovación. Recuperado de http://www.anii.org.uy/web/sites/default/ files/files/No3.pdf

Arnold, M. (1998). Introducción a los Conceptos Básicos de la Teoría General de Sistemas. Cinta de Moebio. Revista de Epistemología de Ciencias Sociales, (3): 40-49 Recuperado de http://www.cintademoebio.uchile.cl/index.php/CDM/ article/view/26455/27748

Baeza, R.. (2006). Jóvenes universitarios chilenos actuales y transformaciones culturales. Estudio sobre nuevas percepciones y prácticas. Revista Sociedad Hoy, (11): 9-33 Recuperado de http://www.redalyc.org/pdf/902/90201102.pdf

Bordieu, P. (1988) La distinción. Buenos Aires: Taurus

Cáceres, F. \& Aceytuno, M. (2008). La innovación como fuente de oportunidades empresariales. Revista de Economía Mundial, (19): 135-156. Recuperado de http://www.redalyc.org/ articulo.oa?id=86601907

Cohen, W. \& Levin, R. (1989). Handbook of Industrial organization. En R. Schmalensee \& R. Willig (Ed), Empirical studies of innovation and Market structure (pp. 1059-1107).http:// dx.doi.org/10.1016/S1573-448X(89)02006-6

Comisión regional de competitividad del Meta. (2008). Plan Regional de Competitividad del Meta. Recuperado de www. comisionesregionales.gov.co/descargar.php?id=61342

Coronado, M., Echeverri, A. \& Arias P. (2014). Aproximación a la cooperación en innovación en empresas del programa de asociatividad y desarrollo empresarial sectorial -PADES- en
Antioquia (Colombia). Revista Facultad De Ciencias Económicas, 22 (2): 185-205. http://dx.doi.org/10.18359/rfce.633

Corporación de Fomento de la Producción CORFO. (2013). Estudio base de innovación percepciones y actitudes - segmento de personas segunda ola diciembre. Recuperado de http://www.innovacion.gob.cl/wp-content/uploads/2014/02/ Percepcion-Innovacion-Personas-2013.pdf

David, F. (1998). La gerencia estratégica. Bogotá: Legis

Duque, E. (2008). Marketing una evidencia cultural. Bogotá: Ecoe

East, R., Harris, P. \& Hammond, K. (1995). Correlates of firstbrand loyalty. Journal of Marketing Management, 11 (5): 487-497. Recuperado de http://doi.org/10.1080/02672 57X.1995.9964360

East, R. (1997). Consumer behaviour advances and applications in marketing, London Prentice Hall.

Helfat, C., Finkelstein, S., Mitchell, W., Peteraf, M., Singh, H., Teece, D., \& Winter, S. (2007). Dynamic Capabilities: Understanding Strategic Change in Organizations. Singapore: Blackwell Publishing.

Jiménez, M. \& Ramos, S. (2007) Jóvenes y móviles. Estrategias de los operadores de telefonía en España. Revista Comunicar, 15 (29): 121-128

Kalish, I. (2008). El Futuro de las Compañías de Productos de Consumo: definiendo el reto, encontrando soluciones. México: Deloitte Services LP. Recuperado de http://www. deloitte.com/assets/Dcom-Panama/Local\%20Assets/Documents/consumo.pdf

Kamakura, W., Kossar, B. \& Wedel, M. (2004). Identifying innovators for the cross-selling of new products. Management Science, 50 (8): 1120-1133. Recuperado de http://search. proquest.com/docview/213186243?accountid=14777

Kuster, B., Aldas M., Rodríguez, S. \& Vila, N. (2010). Diferencias cross-culturales en la decisión de compra de los jóvenes europeos. Revista innovar de ciencias administrativas y sociales, 20 (37): 163-178. Recuperado de http://www.redalyc.org/ articulo.oa?id=81818989013

Luna A., Puello, A. \& Botero, M. (2004). La compra impulsiva y el materialismo en los jóvenes: estudio exploratorio en estudiantes universitarios de Barranquilla (Colombia). Revista Psicología desde el Caribe, (14): 1-26. Recuperado de http:// rcientificas.uninorte.edu.co/index.php/psicologia/article/ viewFile/1777/1157

Lundvall, B. (1992). National Systems of Innovation - Toward a Theory of Innovation and Interactive Learning. Londres: Printer Publishers.

Malaver, F. \& Vargas, M. (2004). Los procesos de innovación en la industria Colombiana: Resultados de un estudio de casos. Revista Cuadernos de Administración, 17 (28): 9-42. Recuperado de http://www.javeriana.edu.co/Facultades/C_Econom_y_Admon/cuadernos_admon/pdfs/1_28.pdf

Moreno R, \& García C. (2014). Sistema para la evaluación de capacidades de innovación en pymes de países en desarrollo: caso Panamá. Revista Facultad De Ciencias Económicas, 22 (2): 109-122. http://dx.doi.org/10.18359/rfce.629

Nelson, R. (1993). National innovation systems: a comparative analysis. Oxford Univ. Press, New York. USA. 
Nelson, R. (1997). Understanding technical change as an evolutionary process. North Holland. Elsevier Science Publishers, USA.

Oliver, R. (1999). Whence consumer loyalty? Journal of Marketing, 63: 33-44 Recuperado de http://www.jstor.org/ stable/1252099.

Organización de cooperación y desarrollo económicos (OCDE), Oficina de estadísticas de las comunidades europeas (2006). Manual de Oslo. Guía para la recogida e interpretación de datos sobre la innovación. Recuperado de http://www.uis. unesco.org/Library/Documents/OECDOsloManual05_spa.pdf

Otero, M. \& Giraldo, W. (2014). La experiencia tecnológica de los niños en su rol de consumidores. Tenth International Congress of Qualitative Inquiry. University of Illinois, UrbanaChampaign, Mayo.

Park, C., Jaworski, B. \& Mcinnis, D. (October, 1986). Strategic Brand Concept-image Management. Journal of Marketing,
50: 135-145 Recuperado de http://www.jstor.org/stable/1251291

Ries, A. \& Trout, J. (1992). Posicionamiento. Juárez: McGraw Hill Schumpeter, J. (1939). The theory of economic development. Harvard. USA.

Schumpeter, J. (1946). Capitalismo, socialismo y democracia. Buenos Aires. Claridad.

Schumpeter, J. (1968).Ensayos. Barcelona. Oikos.

Silverstein, M. (2007). A la caza del tesoro. Barcelona: Urano ediciones.

Valdés, L. (2002). La re-evolución empresarial del siglo XXI. Bogotá: Norma.

Wood, L. (2004). Dimensions of brand purchasing behavior: consumers in the 18-24 age group. Journal of Consumer Behavior, 4 (1): 9-24. Recuperado de https://www.researchgate.net/ publication/227802663. 